Pramāṇa-J. Phys., Vol. 29, No. 4, October 1987, pp. 341-344. (C) Printed in India.

\title{
A note on traversal time for tunneling
}

\author{
A M JAYANNAVAR \\ Department of Physics, Indian Institute of Science, Bangalore 560012, India \\ MS received 12 May 1987
}

\begin{abstract}
A simple approach to study the traversal time for tunneling is given. By using the WKB wave function to evaluate the velocity field of particles in the barrier region, an expression for the traversal time $\tau=\int \mathrm{d} x[m / 2(V(x)-E)]^{1 / 2}$ is obtained in conformity with the recent results.
\end{abstract}

Keywords. Tunneling: traversal time; wave function.

PACS Nos 73.40; 74.50

\section{Introduction}

The phenomenon of particle tunneling through a potential barrier is frequently encountered in physics and has become an important tool for studying the properties of semiconductors, metals and superconductors. Classically a particle whose energy is less than the height of a potential barrier can never pass through it. However, quantum mechanically once an electron is represented by a wave function it penetrates into the classically forbidden region and can tunnel through a potential barrier even though it does not have enough energy to pass over it. In every text book on quantum mechanics one deals with quantum tunneling in the context of, say, a-decay or tunneling of electrons out of a metal surface. In such a situation, one evaluates essentially the transmission coefficient. However, the duration of the actual tunneling process or the time taken by the particle to traverse the barrier has never, been discussed. This problem, however, has been debated in the literature during the past few decades with widely divergent viewpoints (Wigner 1955;Hartman 1962; Smith 1960;Goldberger and Watson 1975;Jauch and Marchand 1967;Rybachenko 1967; Kane 1969;Buttiker and Landauer 1982;Buttiker 1983). Only recently, several workers (Buttiker 1983; Buttiker and Landauer 1982, 1985a, b; Stevens 1983; Jonson 1980; Pollak and Miller 1984; Bruinsma and Bak 1986;Jayannavar 1984)have arrived at the same answer using many different methods, and have also pointed out the unphysical nature of the earlier results. Lately, it has been realized that many phenomena in physics are very sensitive to the traversal time of tunneling. For example, (i) in macroscopic quantum tunneling (Caldeira and Leggett 1981; Voss and Webb 1981; Jayannavar 1985) the tunneling variable is coupled to the reservoir (oscillator) degrees of freedom which results in dissipation. In this case one is interested in the response of the reservoir to the progress of the tunneling event. (ii) In the field emission of electrons from metal surfaces (Jonson 
1980; Schnupp 1968; Hartstein et al 1982; Haavig and Reifenberger 1982), the force exerted by the image charge on the tunneling particle depends on the speed of the tunneling process. Some other examples include resonant tunneling in solids (Ricco and Azbel 1984) and energy exchanges during the tunneling processes that occur in fusion and chemical reactions.

To determine the traversal time for tunneling, Buttiker and Landauer (1982) have considered the tunneling of a particle through a time modulated rectangular potential barrier $V(x, \mathrm{t})=V_{0}(x)+V_{1}(x) \cos (\omega t)$. Now if the period of modulation $\omega^{-1}$ is large compared to the traversal time, then the particle sees an effective static potential barrier. If the period of modulation is small compared to the traversal time then the traversing particle experiences many cycles of oscillation of the potential. In this case, the particle tunnels through a time averaged potential $V_{0}(x)$ and absorbs or emits the modulation quanta. By studying the cross-over between these two types of distinct behaviour they have obtained an expression for $\tau$ which is $\tau=\int_{x_{1}}^{x_{2}}[m / \hbar k(x)] \mathrm{d} x$, where $x_{1}$ and $x_{2}$ are the classical turning points and $k(x)=\left\{2 m\left[V_{0}(x)-E\right]\right\}^{1 / 2} / \hbar$. Buttiker (1983) also has arrived at a similar expression for $\tau$ by studying the extent of Larmor precession that occurs during the transmission of a particle with spin through a rectangular barrier in the presence of a magnetic field (which is confined to the barrier region). For several other approaches see Jonson 1980; Stevens 1983; Pollak and Miller 1984; Bruinsma and Bak 1986.

\section{Formulation of the problem}

In this note we will give a much simpler treatment of the problem as compared to earlier ones, starting from first principles. We deal here with tunneling of particles in the presence of just a static potential barrier. We will make use of the WKB wave function $\psi(x)$ inside the barrier region. This wave function is a linear combination of exponentially growing and decaying wave functions which separately carry zero particle current, but which in combination, due to the complex coefficient, carry a net particle current given by $j(x)=(\hbar / 2 m i)\left[\psi^{*}(\mathrm{~d} \psi / \mathrm{d} x)-\psi\left(\mathrm{d} \psi^{*} / \mathrm{d} x\right)\right]$. The particle current density can be written as (Bohm 1951) $j(x)=v(x) P(x)$, where $P(x)=\psi(x) \psi^{*}(x)$ is the particle density and $v(x)$ is the total velocity field. We will first evaluate the velocity field inside the barrier region $v(x)=[j(x) / P(x)]$, and from this we obtain an expression for $\tau$ which we find to be $\tau=\int_{b}^{a}\left(m / 2[V(x)-E]^{1 / 2}\right) \mathrm{d} x$, where $a$ and $b$ are classical turning points.

Consider a barrier as shown in figure 1 the energy being such that the classical

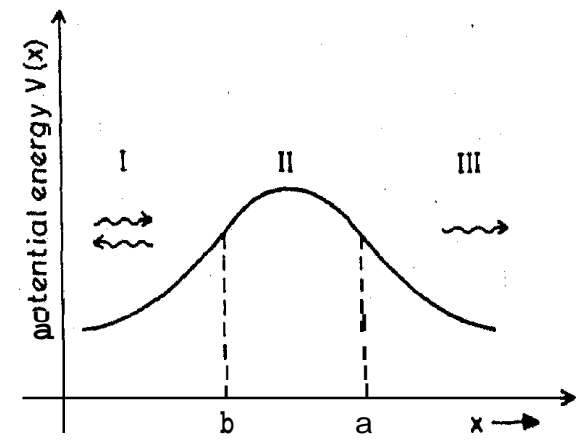

Figure 1. Schematic diagram of the potential barrier, $\boldsymbol{a}$ and $b$ are turning points. 
turning points are at $x=a$ and $\mathrm{x}=b$. The particles are incident from the left (region I); some are reflected and some transmitted. To the right of region III, we have only a transmitted wave, whereas in the region I we have both transmitted and reflected waves. Applying the connection formula the WKB wave function in the barrier region is given by (Bohm 1951).

$$
\psi(x)=\frac{A}{(p(x))^{1 / 2}}\left[\frac{1}{2} \exp \left(-\int_{x}^{a} \frac{p(x)}{\hbar} \mathrm{d} x\right)-i \exp \left(\int_{x}^{a} \frac{p(x)}{\hbar} \mathrm{d} x\right)\right]
$$

where $p(x)=[2 m(V(x)-E)]^{1 / 2}$ and $A$ is a complex number. Given this wave funct: ${ }^{\circ}$ on we evaluate directly the velocity field inside the barrier region and obtain

$$
v(x)=\frac{p(x)}{m}\left[\frac{1}{4} \exp \left(-2 \int_{x}^{a} \frac{p(x)}{\hbar} \mathrm{d} x\right)+\exp \left(2 \int_{x}^{a} \frac{p(x)}{\hbar} \mathrm{d} x\right)\right] .
$$

The WKB approximation is valid slightly away from the turning points. If $\mathrm{x}$ is a point inside the barrier region then the WKB approximation amounts to $\int p(x) \mathrm{d} x / \hbar \gg 1$, so that the negative exponential may be neglected in (23in comparison with the positive exponential (Bohm 1951). This gives

$$
v(x)=\frac{p(x)}{m} \exp \left(-2 \int_{x}^{a} p(x) \frac{\mathrm{d} x}{\hbar}\right)
$$

\section{Discussion}

This is our final result for the total velocity field inside the barrier region. Note that this is a real quantity. Through a semiclassical interpretation of (3) we can obtain the desired result for the traversal time. We will restrict ourselves to the barrier region in the following discussion. The total velocity field at any point $x$ can be written as the vector sum of both the right moving and the left moving particles. Initially particles are incident on the barrier from left and are moving towards right. In the barrier region, the right moving particles have a forward velocity $[p(x) / m]$. At the point $\mathrm{x}$, the particles moving to right will experience the potential barrier extending from $x$ to a. Consequently some of them will be reflected with the reflection coefficient $R(x)$ (there are no multiple reflections). Hence the total velocity field at the point $x$ is given by $v(x)=[(p(x) / m)-R(x)(p(x) / m)]=T(x)(p(x) / m)$, where $T(x)$ is the transmission coefficient for the barrier which extends from $x$ to a. Now the exponential term in (3) is nothing but the WKB expression for the transmission coefficient (Bohm 1951), if the barrier extends only from $x$ to $a$. This naturally implies that if the particles are finally transmitted they would have travelled with forward velocity $[p(x) / m]$, with no multiple reflections within the barrier region. Hence the traversal time for tunneling is given by $\tau=\int_{b}^{a}[\mathrm{~m} / p(x)] \mathrm{d} x$, the same expression as that obtained by several authors. There is another simple way of seeing that the forward velocity of particles is $[p(x) / m]$. Let us take the extreme quantum limit, i.e. $\hbar \rightarrow \infty$, of (3); we then get $v(x)=[p(x) / m]$. In this limit, the particles impinging on the barrier are transmitted through it with unit probability. Therefore the total velocity field is nothing but the forward moving velocity, which is $[p(x) / m]$. 
We wish to point out that the form of $v(x)$ in (3) naturally leads to a simple interpretation in the form of statistical composition law for the velocity field without any quantum interference terms. This is probably due to the very nature of the WKB wave function in the barrier region. We have shown that, quantum mechanically a real velocity is obtained for a particle in a classically forbidden region. This velocity coincides with that of a classical particle moving in the euclidean potential $(-V(x))$ with energy $(-\boldsymbol{E})$.For the simple case of the rectangular potential barrier $V_{0}$ (thickness d) and the inverted parabolic barrier $V(x)=\left(-\omega_{0}^{2} x^{2} / 2\right)$, an explicit expression for $\tau$ is $\left[m / 2\left(V_{0}-E\right)\right]^{1 / 2} d$ and $\left(\pi \sqrt{m} / \omega_{0}\right)$, respectively. For the explicit calculation of the traversal time for other physically interesting potential barriers, see Buttiker and Landauer 1985 b.

\section{References}

Bohm D 1951 Quantum theory (New Jersey: Prentice Hall)

Bruinsma R and Bak P 1986 Phys. Rev. Lett. 56420

Buttiker M 1983 Phys. Rev. B27 5178

Buttiker M and Landauer R 1982 Phys. Rev. Lett. 491739

Buttiker M and Landauer R 1985a Phys. Scr. 32429

Buttiker M and Landauer R 1985b Adv. Solid State Phys. (ed) P Grosse 25711

Caldeira A O and Leggett A J 1981 Phys. Rev. Lett. 46211

Gsldberger M L and Watson K M 1975 Collision Theory (Huntington N.Y: Krieger).

Haavig D L and Reifenberger R 1982 Phys. Rev. B26 6408

Hartman T E 1962 J. Appl. Phys. 333427

Hartstein A, Weinberg Z A and Di Maria D J 1982 Phys. Rev. B25 7174

Jauch J M and Marchand J P 1967 Phys. Acta 40217

Jayannavar A M 1984 ICTP Preprint IC/84/49

Jayannavar A M 1985 Lett. Nuovo Cimento 42141

Jonson M 1980 Solid State Commun. 33743

Kane E O 1969Tunneling phenomena in solids(Eds.)E Burstein and S Lundqvist (New York: Plenum Press)

Pollak E and Miller W H 1984 Phys. Rev. Lett. 53115

Ricco B and Azbel M Ya 1984 Phys. Rev. B29 1970

Rybachenko V F 1967 Sov. J. Nucl. Phys. 5365

Schnupp P 1968 Thin solid films 2177

Smith F T 1960 Phys. Rev. 118349

Stevens K W H 1983 J. Phys. C16 3649

Voss R F and Webb R A 1981 Phys. Rev. Lett. 47265

Wigner E P 1955 Phys. Rev. 98145 